University of Nebraska - Lincoln

DigitalCommons@University of Nebraska - Lincoln

Genetic Consequences of Polygyny and Social Structure in an Indian Fruit Bat, Cynopterus sphinx. I. Inbreeding, Outbreeding, and Population Subdivision

\author{
Jay F. Storz \\ University of Nebraska - Lincoln, jstorz2@unl.edu \\ Hari Bhat \\ National Institute of Virology, Pune, 411 001, India (Present address: 107 Awanti, OPP: Kamala Nehru \\ Park, Erandawana, Pune, 411 004, India) \\ Thomas Kunz \\ Department of Biology, Boston University, 5 Cummington Street, Boston, Massachusetts
}

Follow this and additional works at: https://digitalcommons.unl.edu/bioscistorz

Part of the Genetics and Genomics Commons

Storz, Jay F.; Bhat, Hari; and Kunz, Thomas, "Genetic Consequences of Polygyny and Social Structure in an Indian Fruit Bat, Cynopterus sphinx. I. Inbreeding, Outbreeding, and Population Subdivision" (2001). Jay F. Storz Publications. 21.

https://digitalcommons.unl.edu/bioscistorz/21

This Article is brought to you for free and open access by the Papers in the Biological Sciences at DigitalCommons@University of Nebraska - Lincoln. It has been accepted for inclusion in Jay F. Storz Publications by an authorized administrator of DigitalCommons@University of Nebraska - Lincoln. 


\title{
Genetic Consequences of Polygyny and Social Structure in an Indian Fruit Bat, Cynopterus sphinx. I. Inbreeding, Outbreeding, and Population Subdivision
}

\author{
Jay F. Storz, ${ }^{1}$ Hari R. Bhat, ${ }^{2}$ and Thomas H. Kunz ${ }^{1}$ \\ ${ }^{1}$ Department of Biology, Boston University, 5 Cummington Street, Boston, Massachusetts 02215, \\ ${ }^{2}$ National Institute of Virology, Pune, 411 001, India (Present address: 107 Awanti, OPP: Kamala Nehru Park, Erandawana, Pune, 411 004, India)
}

\begin{abstract}
Population subdivision into behaviorally cohesive kin groups influences rates of inbreeding and genetic drift and has important implications for the evolution of social behavior. Here we report the results of a study designed to test the hypothesis that harem social structure promotes inbreeding and genetic subdivision in a population with overlapping generations. Genetic consequences of harem social structure were investigated in a natural population of a highly polygynous fruit bat, Cynopterus sphinx (Chiroptera: Pteropodidae), in western India. The partitioning of genetic variance within and among breeding groups was assessed using 10-locus microsatellite genotypes for 431 individually marked bats. Genetic analysis of the C. sphinx study population was integrated with field data on demography and social structure to determine the specific ways in which mating, dispersal, and new social group formation influenced population genetic structure. Microsatellite data revealed striking contrasts in genetic structure between consecutive offspring cohorts and between generations. Relative to the 1998 (dry-season) offspring cohort, the 1997 (wet-season) cohort was characterized by a more extensive degree of withingroup heterozygote excess $\left(F_{\mathrm{IS}}=-0.164\right.$ vs. -0.050$)$, a greater degree of among-group subdivision $\left(F_{\mathrm{ST}}=0.123 \mathrm{vs}\right.$. 0.008$)$, and higher average within-group relatedness ( $r=0.251$ vs. 0.017). Differences in genetic structure between the two offspring cohorts were attributable to seasonal differences in the number and proportional representation of male parents. Relative to adult age-classes, offspring cohorts were characterized by more extensive departures from allelic and genotypic equilibria and a greater degree of genetic subdivision. Generational differences in F-statistics indicated that genetic structuring of offspring cohorts was randomized by natal dispersal prior to recruitment into the breeding population. Low relatedness among harem females $(r=0.002-0.005)$ was primarily attributable to high rates of natal dispersal and low rates of juvenile survivorship. Kin selection is therefore an unlikely explanation for the formation and maintenance of behaviorally cohesive breeding groups in this highly social mammal.
\end{abstract}

Keywords: F-statistics, inbreeding, mating system, metapopulation, microsatellite DNA, population structure, relatedness

Population subdivision plays a potentially important role in processes of both adaptive and nonadaptive evolutionary change. The partitioning of genetic variance within and among different hierarchical levels of population structure influences the genetically effective size of the total population $\left(N_{e}\right)$ and the extent to which different components of genetic variance are made available to selection (Barton and Whitlock 1997; Wade and Goodnight 1998; Nunney 1999). In populations that are subdivided into socially defined breeding units, the evolution of cooperative and altruistic behaviors is of particular interest. Theory indicates that some form of subdivision is required for the initial spread and maintenance of such traits (Uyenoyama 1984; Wade and Breden 1987; Michod 1993; Wade 1996). Regardless of whether population genetic structure constitutes a primary cause or secondary consequence of sociality, the subdivision of populations into stable kin groups has important implications for the course of microevolutionary events.

Populations of many mammalian species are subdivided into behaviorally segregated breeding groups maintained by sex-biased philopatry and territorial exclusion of immigrants (Greenwood 1980; Dobson 1982; Chepko-Sade and Shields 1987; Pusey 1987; Pusey and Packer 1987; Clutton-Brock 1989). These socially mediated constraints on gene flow, in combination with polygynous mating, may promote a substantial degree of genetic subdivision among breeding groups (Chesser 1991a,b; Storz 1999). Genetic consequences of social structure can be analyzed within the framework of Wright's $(1951,1965)$ hierarchical F-statistics. With reference to socially defined levels of population structure, $F$-statistics are defined as follows: $F_{\mathrm{IS}}$-values represent allelic correlations within individuals relative to breeding groups; $F_{\mathrm{IT}}$-values represent allelic correlations within individuals relative to the total population; and $\mathrm{FST}^{-}$ values represent allelic correlations within breeding groups relative to the total population. Because expectations for the set of F-statistics depend on population age structure, a sampling design that delineates consecutive generations provides the greatest scope for making inferences about population breeding structure. Departures from HardyWeinberg genotypic proportions, as revealed by $F_{\mathrm{IS}}$ and $F_{\mathrm{IT}}$, can be used to identify the patterning and magnitude of barriers to random mating.

In discussions of topics ranging from dispersal ecology (Chepko-Sade and Shields 1987) to chromosomal evolution (Wilson et al. 1975; Bush et al. 1977), populations of social mammals are commonly envisioned as networks of partially isolated breeding groups characterized by small $\mathrm{N}_{\mathrm{e}^{-}}$ values and relatively high levels of inbreeding. Such a view predicts the general occurrence of high $F_{\mathrm{ST}}$ among groups (due to the combined effects of small $N_{\mathrm{e}}$-values and low rates of intergroup dispersal) and positive $F_{\text {IS }}$-values (due to inbreeding within closed social groups). However, the 
picture that emerges from a review of theoretical and empirical findings (Storz 1999) suggests that social units of mammalian populations do not closely approximate the inbred isolates envisioned by many previous workers. In populations of social mammals, genetic subdivision among breeding groups is typically accompanied by strongly negative $F_{\mathrm{IS}}$-values, indicating a within-group excess of heterozygotes relative to Hardy-Weinberg expectations. This conclusion was anticipated by the results of theoretical models of population structure that incorporated the effects of sex differences in dispersal and variance in reproductive success (Chesser 1991a,b; Chesser et al. 1993; Wang 1997).

The objective of this study was to test the hypothesis that harem social structure promotes inbreeding and genetic subdivision in a population with overlapping generations. This hypothesis was tested by investigating the genetic consequences of social structure in a natural population of the short-nosed fruit bat, Cynopterus sphinx (Chiroptera: Pteropodidae), in western India. To determine the specific ways in which mating, dispersal, and new group formation influence population genetic structure, genetic analysis of the $C$. sphinx study population was integrated with field data on demographic population structure (Storz et al. 2000b).

\section{Materials and Methods}

\section{Study Population}

Cynopterus sphinx is a harem-forming frugivorous bat that is widely distributed across tropical southern Asia (Storz and Kunz 1999; Storz et al. 2001a). Cynopterus sphinx makes use of a wide variety of diurnal roosts and is known to alter different types of foliage to construct "tents" (Balasingh et al. 1995; Bhat and Kunz 1995; Storz et al. 2000a,b). In our study site in western India (Pune, Maharashtra, $\left.18^{\circ} 32^{\prime} \mathrm{N}, 73^{\circ} 51^{\prime} \mathrm{E}\right)$, harem breeding groups of $C$. sphinx roost in tents constructed within flower/fruit clusters of the kitul palm (Caryota urens; Bhat and Kunz 1995; Storz et al. 2000b). The breeding population of $C$. sphinx in Pune is subdivided into diurnal roosting colonies, each containing one to five harems and often one or more satellite males in adjacent roosts. Colonies comprise all bats occupying flower/fruit cluster tents in a single tree or cluster of two or three adjacent trees. In many cases, there is only one tent-roosting harem per colony. In other colonies, several harems occupy different tents in the same or adjacent trees. A given tree may have as many as five flower/fruit clusters at one time, although only one new flower cluster will become available for tent construction per season. Thus, when two or more tents are occupied in the same tree, the potential discrepancy in age (time since founding) between resident harems varies between one and four years (Storz et al. 2000b).

Harems consist of a single adult male roosting in association with one to 37 reproductive females and their dependent young. Harem size averages 6.1 adults in the wet season $(\mathrm{SD}=3.5)$ and 13.6 adults in the dry season $(\mathrm{SD}=8.5)$. The same harem social configuration is maintained yearround, despite a high degree of synchrony and seasonality in the timing of reproduction. Mating opportunities are monopolized by a relatively small number of territorial males, such that seasonal offspring cohorts are largely composed of paternal sibships that range in size from two to 20 pups (Storz et al. 2001b). Adult females often remain associated as roostmates from one parturition period to the next, and group cohesion is unaffected by turnover of harem males. Females frequently transfer among roosts within the same colony, and harems undergo periodic fissions and fusions. Juveniles of both sexes disperse after weaning, and sexually immature bats are never present in harems at the time of parturition (Storz et al. 2000a,b).

\section{Sampling}

Complete harem groups and solitary males were trapped in their diurnal roosts, and pups and adults were individually marked (see Storz et al. 2000a,b). Bats were sampled over a period of approximately four weeks immediately following each of two annual parturition periods: July-August 1997 (wet season) and March-April 1998 (dry season). The breakdown by collection was as follows: 1997 wet season, 14 harems from nine colonies (81 adult females and 67 pups); 1998 dry season, 13 harems from 10 colonies (153 adult females and 118 pups). Additionally, we sampled all males that defended territories within the study area over a two-year period (1996-1998) that spanned the dates of conception of sampled pups. Bats were sampled when nearly all females had given birth but pups had not yet been weaned. Most pups were two to three weeks old at the time of sampling, and all were matched with known mothers. For all pups and adults, biopsy punches (4-mm diameter) were taken from the patagial membrane and stored in a $20 \% \mathrm{DMSO} / 5 \mathrm{M} \mathrm{NaCl}$ solution $(\mathrm{pH}=7.5)$ prior to DNA extraction. Bats were held in net cages and released at their roosts on the evening of the same day they were captured. A total of 431 individually marked bats were sampled for the genetic analysis (209 females, 185 suckling pups, and 37 territory-holding males).

\section{Screening Microsatellite DNA Variation}

Genomic DNA was isolated from tissue samples of $C$. sphinx using QIAamp extraction columns (Qiagen Inc., Valencia, CA). Bats were genotyped at a total of 10 microsatellite loci: one di-, three tri-, and six tetranucleotide repeat polymorphisms. Primer sequences and polymerase chain reaction (PCR) protocols for tri- and tetranucleotide repeat loci were reported in Storz (2000). Using the same PCR protocol used to amplify CSP-1, CSP-5, and CSP-6 (see Storz 2000), the dinucleotide repeat locus (locus 3) was amplified with the following primer pair: 5'-CTCTAACTTGCTCCTTCAGC-3' and 5'-CTACTAAATGACGCCTCTACC-3' (G. F. McCracken, unpubl. ms.). Allele sizes were quantified using a 377 ABI Prism automated sequencer and analyzed using GENESCAN software (PE Applied Biosystems, Foster City, CA).

Assessment of Population Genetic Structure: Tests of Disequilibrium and Genetic Subdivision

While treating parental and filial generations separately, linkage disequilibrium was tested using an exact probability test for $\mathrm{R} \times \mathrm{C}$ contingency tables (Raymond and Rous- 
Table 1. Alternative hypotheses regarding inbreeding, outbreeding, and the interplay between patterns of mating and dispersal in a tent-roosting population of Cynopterus sphinx in Pune, India. See text for explanation of parameter symbols and the underlying rationale for each hypothesis.

Mating/dispersal pattern

Polygyny coupled with positive assortative mating (inbreeding)

Polygyny coupled with negative assortative mating (outbreeding)

Polygyny coupled with female philopatry

Polygyny coupled with female dispersal
Alternative hypotheses set 1995a). Unbiased estimates of exact probabilities were obtained using the Markov chain algorithm of Guo and Thompson (1992), as implemented in the program GENEPOP 3.1d (Raymond and Rousset 1995b). Compared to tests of Hardy-Weinberg equilibrium (HWE), linkage disequilibrium is a more sensitive measure of the effects of nonrandom mating because a population will return to HWE after a single generation of random mating (when generations are nonoverlapping) or a relatively small number of generations (when generations overlap). In contrast, linkage disequilibrium is dissipated at a much slower rate determined by the recombination fraction (Hartl and Clark 1997).

Generations were treated separately, such that genetic structuring was assessed for the same groups composed of adult females only and pups only. For each generation, only groups composed of four or more individuals were included in the analysis. Partitioning of genetic variance within and among breeding groups was assessed using Wright's (1951, 1965) F-statistics. Specifically, we used the estimators of Weir and Cockerham (1984): $f\left(F_{\text {IS }}\right), F\left(F_{\text {IT }}\right)$, and $\theta\left(F_{\mathrm{ST}}\right)$. Null hypotheses of Hardy-Weinberg genotypic proportions $\left(F_{\mathrm{IS}}=0\right.$ and $F_{\mathrm{IT}}=0$ ) were tested by means of a randomization procedure. Null distributions were generated from 10,000 randomizations of alleles among individuals within groups (for $F_{\text {IS }}$ ) and among individuals sampled across the entire population (for $F_{\mathrm{IT}}$ ). Because different forms of nonrandom mating are expected to cause deviations from Hardy-Weinberg proportions in opposite directions (inbreeding produces a heterozygote deficit, whereas variance in mating success may produce a heterozygote excess), two-tailed probability tests were used to assess statistical significance of observed values relative to null distributions. For $F_{\mathrm{ST}}$, bootstrap resampling across loci was used to obtain $95 \%$ and $99 \%$ confidence intervals, calculated as the range of values that bracketed the corresponding percentage of bootstrap estimates. Genetic subdivision among groups was also tested using an exact G-test of genotypic frequencies (Goudet et al. 1996). A total of 10,000 randomizations (permuting genotypes across groups) was used to generate a probability distribution under the null hypothesis of no differentiation. The program FSTAT 2.8 (Goudet 1995) was used for all calculations.

\section{Genetic Consequences of Mating, Dispersal, and New Group Formation}

Genetic consequences of social structure were inferred by testing a set of hypotheses formulated in terms of generation-specific expectations for F-statistics (Table 1). Inbreeding (positive assortative mating with respect to kinship) is expected to produce a heterozyogote deficit relative to HWE, and correlation between united gametes in progeny $\left(F_{\mathrm{IT}}[\mathrm{offspring}]\right)$ is expected to be similar to that between random gametes in the parental generation ( $F_{\mathrm{ST}}$ [adults]). Conversely, systematic avoidance of consanguineous mating (negative assortative mating with respect to kinship) is expected to produce a heterozygote excess relative to HWE, and $F_{\mathrm{ST}}$ (adults) is expected to exceed $F_{\mathrm{IT}}$ (offspring). When females are philopatric, such that harems constitute matrilineal kin groups, $F_{\mathrm{ST}}$ is expected to be similar in maternal and filial generations. In contrast, a generational disparity in potential coancestry results when juvenile females are not recruited into natal breeding groups. In harem-forming species characterized by female dispersal (and a litter size of one), same-age offspring may often represent the progeny of a single male and multiple females, whereas harem females represent the progeny of multiple males and multiple females. Under such circumstances, $F_{\mathrm{ST}}$ is expected to be greater in offspring relative to adult females.

To assess the effects of group turnover on population genetic structure, we investigated the relative degree of genetic subdivision among harems representing different age classes (different times since founding). Age of harems was determined by the maximum potential number of parturition periods that had elapsed (at the time of sampling) since the group was initially founded (Storz et al. 2000b). Harems were considered newly founded if the harem was sampled immediately following the first parturition period that occurred in a particular roost. Harems were considered previously established if two or more reproductive cycles had elapsed since the roost was originally colonized. Because group turnover is expected to enhance genetic subdivision when variance among newly founded groups is greater than that among preexisting groups at genetic equilibrium (Whitlock and McCauley 1990; Whitlock 1992), we tested the null hypothesis $F_{\mathrm{ST}}$ (newly founded) $=F_{\mathrm{ST}}$ (previously established). Because only one harem was newly founded in the 1997 wet season, this test was performed only for harems in the 1998 dry season when newly founded and previously established harems were equal in number (Storz et al. 2000b). The test was restricted to harems composed of at least four adult females.

Estimating relatedness - The coefficient of genetic relatedness ( $r$ ) among pups and adults was estimated according to the method of Queller and Goodnight (1989). Single-generation pedigree links in each offspring cohort were previously established by means of genetic parentage analysis (Storz et al. 2001b). We therefore estimated symmetrical $r$ - values for known mother-offspring, father-offspring, and half-sibling dyads to provide a calibration for pairwise 
Table 2. Summary statistics for microsatellite DNA markers used in the assessment of population genetic structure in Cynopterus sphinx. $N$, number of bats genotyped per locus; $N_{\mathrm{A}^{\prime}}$ number of alleles per locus; $H_{\mathrm{O}^{\prime}}$ observed heterozygosity; and $H_{\mathrm{E}^{\prime}}$ expected heterozygosity.

\begin{tabular}{lccrcc}
\multicolumn{1}{c}{$\begin{array}{c}\text { Allele } \\
\text { Lize range }\end{array}$} & $N$ & $N_{\mathrm{A}}$ & $H_{\mathrm{O}}$ & $H_{\mathrm{E}}$ \\
Locus 3 & $174-194$ & 431 & 10 & 0.74 & 0.76 \\
CSP-1 & $191-218$ & 431 & 9 & 0.73 & 0.71 \\
CSP-2 & $113-134$ & 431 & 7 & 0.78 & 0.74 \\
CSP-3 & $95-107$ & 431 & 5 & 0.37 & 0.38 \\
CSP-4 & $139-163$ & 431 & 10 & 0.79 & 0.78 \\
CSP-5 & $110-170$ & 431 & 12 & 0.76 & 0.73 \\
CSP-6 & $127-219$ & 431 & 14 & 0.81 & 0.85 \\
CSP-7 & $231-265$ & 431 & 17 & 0.82 & 0.82 \\
CSP-8 & $150-202$ & 420 & 14 & 0.75 & 0.74 \\
CSP-9 & $278-298$ & 431 & 5 & 0.49 & 0.47
\end{tabular}

estimates of average $r$ among pups within each offspring cohort, and all pairwise combinations of adult males and females in the study population. Relatedness coefficients were calculated using the program KINSHIP 1.3 (Goodnight and Queller 1999). In conjunction with estimates of $F$ statistics, we report estimates of average within-group $r$ for both parental and filial generations. We obtained $95 \%$ and $99 \%$ confidence intervals for estimates of average withingroup $r$ by bootstrapping over loci, using the program FSTAT 2.8 (Goudet 1995).

In subdivided populations, the identity-by-descent $(i b d)$ estimator of $r$ can be interpreted within the same analytical framework as the Weir and Cockerham (1984) estimators of F-statistics (Queller and Goodnight 1989). In a subdivided population, average within-group relatedness is estimated as $r=2 F_{\mathrm{ST}} /\left(1+F_{\mathrm{IT}}\right)$ (Wright 1922; Michod 1993). Both estimators use the same method for combining information across loci, but the Queller and Goodnight (1989) method uses a different bias correction for group membership. In the Weir and Cockerham estimation procedure, sums of squares of allelic frequencies are multiplied by a uniform sample-size correction factor. In contrast, the Queller and Goodnight method makes use of group-specific correction factors whereby mean allelic frequencies for the total population are recalculated after omitting each group in turn. $F$-statistics can be interpreted as ratios of actual ibd-val- ues to maximum possible $i b d$ above random expectation. For the fixation indices $F_{\mathrm{IS}}$ and $F_{\mathrm{IT}}$, the $i b d$ of interest is that between alleles at the same locus, scaled according to expected identities at the appropriate hierarchical level of population structure. Different methods of bias correction thus result in slightly different expectations for the baseline level of $i b d$ when groups vary in genotypic composition.

\section{Results}

Tests of Disequilibrium and Genetic Subdivision

Microsatellite loci proved highly informative for the purpose of assessing population genetic structure: observed heterozygosity averaged 0.70 (range $=0.37-0.82$ ) and number of alleles per locus averaged 10.3 (range $=5-17$; Table 2). Using the sequential Bonferroni test with a 0.05 experiment-wise error rate (Sokal and Rohlf 1995), a statistically significant degree of two-locus linkage disequilibrium was indicated for two of 45 pairwise comparisons in the 1997 offspring cohort (CSP-5 and CSP-6, $P<0.0001$; locus 3 and CSP-9, $P=0.0003$ ). No significant departures from linkage equilibria were observed in the 1998 offspring cohort or in the adult female population of either year.

Microsatellite data revealed striking contrasts in genetic structure between consecutive offspring cohorts and between generations in each year of the study. Relative to adult age classes, offspring cohorts were characterized by more extensive departures from Hardy-Weinberg genotypic proportions and a greater degree of among-group subdivision. In both years, offspring cohorts exhibited a within-group excess of heterozygotes relative to HardyWeinberg expectations (Table 3). A significant degree of population-wide heterozygote excess was also indicated in the 1998 offspring cohort. Exact G-tests revealed a significant degree of genotypic subdivision among groups in both cohorts. Relative to the 1998 cohort, offspring in the 1997 cohort were characterized by a more extensive degree of within-group heterozygote excess $\left(F_{\mathrm{IS}}=-0.164 \mathrm{vs}\right.$. $-0.050)$, a greater degree of among-group subdivision $\left(F_{\mathrm{ST}}\right.$ $=0.123$ vs. 0.008$)$, and higher average within-group relatedness ( $r=0.251$ vs. 0.017 ).

With regard to the adult female population, no significant degree of genetic structure was revealed in 1997 (Table 3).

Table 3. Summary statistics describing the partitioning of genetic variance within and among harem breeding groups of Cynopterus sphinx. Standard errors of $F$-statistics and the within-group relatedness coefficient $(r)$ were obtained by jackknifing over loci. Statistical significance of $F_{\text {IS }}$ and $F_{\mathrm{IT}}$ was assessed by a randomization procedure (see text for details). For $F_{\mathrm{ST}}$ and $r, 95 \%$ confidence intervals were obtained by bootstrapping over loci. Results of the exact $G$-test $\left(P_{G}\right)$ are expressed as the proportion of randomizations (permutations of genotypes across groups) that yielded values larger than the observed value.

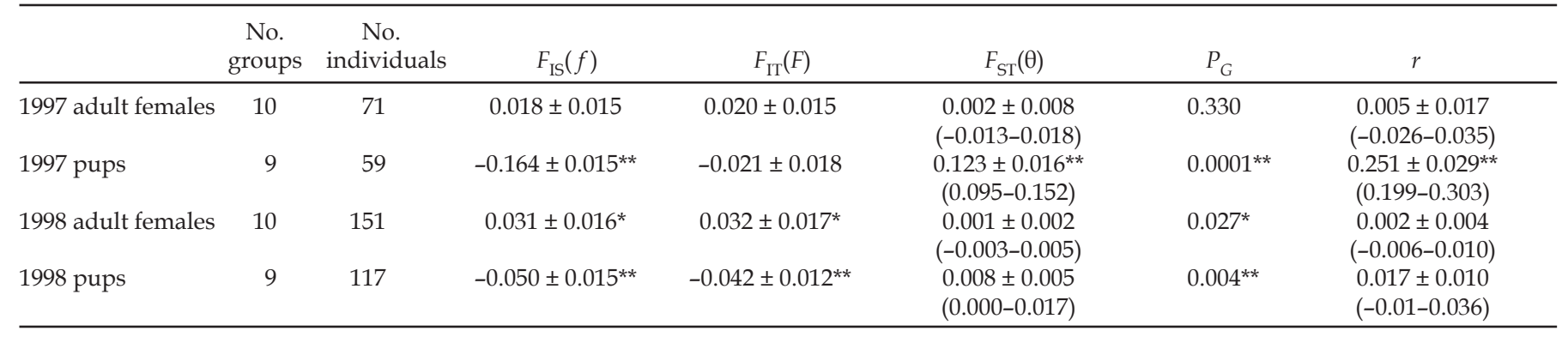

* $P<0.05$; ** $P<0.01$. 
Table 4. Average pairwise relatedness coefficients for individuals of Cynopterus sphinx in different age and sex categories. Parent-offspring and half-sibling relationships were inferred from a genetic analysis of parentage (Storz et al. 2001b).

\begin{tabular}{lccc}
\hline & $\begin{array}{c}\text { Average } \\
\text { pairwise } \\
r\end{array}$ & $\begin{array}{c}95 \% \\
\text { confidence } \\
\text { interval }\end{array}$ & $\begin{array}{r}\text { No. } \\
\text { pairwise } \\
\text { combinations }\end{array}$ \\
\hline Mother-offspring pairs & 0.494 & 0.478 to 0.511 & 185 \\
Father-offspring pairs & 0.508 & 0.485 to 0.530 & 118 \\
Half-siblings & 0.298 & 0.285 to 0.310 & 737 \\
Pups (1997 cohort) & 0.036 & 0.027 to 0.044 & 2211 \\
Pups (1998 cohort) & -0.002 & -0.006 to 0.003 & 6903 \\
Adult females & -0.008 & -0.010 to -0.006 & 21,736 \\
Adult males & -0.001 & -0.014 to 0.013 & 666 \\
\hline
\end{tabular}

In contrast, the adult female population in 1998 was characterized by a marginally significant heterozygote deficit within groups $\left(F_{\mathrm{IS}}=0.031\right)$ in addition to a population-wide deficit $\left(F_{\mathrm{IT}}=0.032\right)$. Moreover, the exact $G$-test revealed a moderate degree of genotypic subdivision among groups. In samples from both generations, bootstrap confidence intervals for $F_{\mathrm{ST}}$ often included zero even when the exact $G$ test indicated a significant degree of genotypic subdivision (Table 3).

Overall levels of relatedness were low within offspring cohorts and among adults of both sexes (Table 4). Only in the 1997 offspring cohort were within-group $r$-values elevated significantly above ambient levels of allele-sharing (mean $r=0.251$; Table 3). Average relatedness among adult males that defended roosts within the same colony was not significantly different from zero $(r=0.024 \pm 0.035,95 \% \mathrm{CI}=$ $-0.040-0.089$ ).

\section{Genetic Consequences of Group Turnover}

Mark-recapture records indicated that the formation of new harems most often resulted from the fissioning of previously cohesive harems within the same colony (Storz et al. 2000b). Some harems contained disproportionate numbers of young females, as indicated by reproductive status and relative age estimates (Storz et al. 2000a,b). This pattern suggests that some groups were founded by members of the same age cohort (e.g., half-sibling progeny of a single harem). However, there was no evidence for kin-structured group formation, as $90 \%$ confidence intervals were broadly overlapping for $F_{\mathrm{ST}}$ (newly founded) $(=0.001)$ and $F_{\mathrm{ST}}$ (previously established) $(=0.002)$.

\section{Discussion}

\section{Population Subdivision}

Interpretation of $F_{S T}-$ Differences in linkage disequilibrium and genetic structure between the two offspring cohorts were attributable to seasonal differences in the number and proportional representation of male parents. Genetic paternity analysis revealed that the majority of harem offspring in the 1997 (wet-season) cohort were sired by the resident harem male (Storz et al. 2001b). This is reflected in the estimate for average $r$ within harems (0.251), which is at the one-quarter level expected for half-siblings.
Offspring born in the wet-season parturition period were conceived 115-135 days prior, during the postpartum estrus period (midway through the dry season) when the level of female aggregation is highest and the potential for polygyny is at its peak (Storz and Kunz 1999; Storz et al. $2000 a, b)$. However, the level of shared paternity among same-age progeny of a single harem reflects the compositional stability of female groups in addition to the degree of male reproductive control. Even if harem males have exclusive mating access to all female roostmates during the estrus period, multiple fathers may be represented among offspring of a single harem if females transfer among harems between conception and parturition. Thus, in addition to seasonal variation in the degree of polygyny, cohort-specific differences in within-group relatedness (and thus $F_{\mathrm{ST}}$ ) also suggest that most intergroup transfers of adult females occur in the interval between the wet-season breeding period and the subsequent dry-season parturition period.

The adult gene pool of C. sphinx was characterized by a generally inconsequential degree of genetic subdivision. Although the exact $G$-test revealed a subtle degree of genotypic differentiation among harems in 1998, average within-harem relatedness $(r=0.002)$ was not significantly greater than zero. In combination with mark-recapture and census data (Storz et al. 2000b) the genetic data indicated that sampling drift associated with polygynous mating is counteracted by the randomizing effects of natal dispersal prior to recruitment into the breeding population. The low degree of genetic subdivision among harems of $C$. sphinx $\left(F_{\mathrm{ST}}=0.001-0.002\right)$ is similar to that observed in other polygynous bats that form harems (Phyllostomus hastatus, $F_{\mathrm{ST}}$ $=0.031$; McCracken and Bradbury 1981; McCracken 1987) or multimale/multifemale breeding groups (Desmodus rotundus, $F_{\mathrm{ST}}=0.049$; Wilkinson 1985b). Contrary to the pattern observed in most group-living social mammals (Clutton-Brock 1989), available evidence for polygynous bats suggests that juveniles of both sexes typically disperse from their natal breeding group before reaching sexual maturity (Bradbury and Vehrencamp 1976; McCracken and Bradbury 1981; Williams 1986; Storz et al. 2000b). Although juvenile females of the common vampire bat (Desmodus rotundus) are recruited into their natal breeding groups, the occasional intergroup exchange of breeding females and low rate of juvenile survivorship does not allow significant levels of matrilineal relatedness to accrue (Wilkinson 1985a,b). Similarly, low relatedness among harem females of C. sphinx ( $r=0.002-0.005)$ is attributable to the complete natal dispersal of juvenile females and low rate of juvenile survivorship (12\% per year; Storz et al. 2000b).

The genetic data revealed no evidence of kin-structured group formation in the C. sphinx study population because there was no significant difference in $F_{\mathrm{ST}}$ between age-classes of harems (newly founded vs. previously established). This contrasts with the dynamics of new group formation in the Yanomama tribe, where lineal fissioning of villages along lines of patrilineal kinship produced a significant degree of genetic subdivision (Smouse et al. 1981).

When units of sampling are small relative to levels of allelic diversity, exact tests based on allelic or genotypic counts appear to be the most powerful means of detecting 
genetic subdivision, especially for unbalanced sampling designs (Raymond and Rousset 1995a; Goudet et al. 1996). This explains why the exact G-test often revealed statistically significant genotypic differentiation among groups even when $95 \%$ bootstrap confidence intervals for unbiased estimates of $F_{\mathrm{ST}}(\theta)$ bracketed zero (Table 3).

\section{Inbreeding and Outbreeding}

Interpretation of $F_{I S^{\circ}}$-In contrast to the striking pattern of heterozygote excess observed in offspring cohorts, the adult female gene pool more closely conformed to panmictic expectations, and slight departures from Hardy-Weinberg proportions in 1998 were in the direction of heterozygote deficit. Departures from Hardy-Weinberg genotypic proportions can result from a number of different demographic factors. Inbreeding is expected to produce a heterozygote deficit in progeny relative to Hardy-Weinberg expectations ( $F_{\text {IS }}$ $>0)$. In contrast, polygynous mating is expected to produce a heterozygote excess in progeny $\left(F_{\mathrm{IS}}<0\right)$ because when the effective number of breeding males is small, frequencies of paternally and maternally contributed alleles will differ due to binomial sampling error. The same effect is produced by differences in allelic frequencies between the sexes.

When females are recruited into natal breeding groups, the potential for inbreeding depends on the frequency with which breeding tenure of males overlaps the onset of sexual maturity of their female progeny and the frequency with which juvenile males attain breeding status within their natal group. Potential levels of inbreeding are thus largely determined by the rate at which males transfer among groups. Inbreeding may be minimized in populations of social mammals either as a direct result of behaviors that promote the active avoidance of incestuous matings or as an indirect outcome of sex-biased dispersal (Pusey 1987; Clutton-Brock 1989; Smith 1993; Pusey and Wolf 1996). Even in the absence of active inbreeding avoidance by means of kin discrimination, negative values of $F_{\mathrm{IS}}$ are to be expected in populations characterized by the typically mammalian pattern of polygynous mating and male-biased dispersal. In a harem-forming species characterized by female philopatry, all or most matings occur between an immigrant male drawn from one matriline and multiple females composing a genetically distinct matriline. Heterozygote excess in the first generation offspring thus results from a Wahlund effect between the sexes. The magnitude of departure from Hardy-Weinberg expectations (in the direction of heterozygote excess) is directly proportional to the genetic variance between matrilines (Prout 1981; Chesser 1991a). Genetic subdivision and within-group heterozygote excess is therefore maximized when single males mate exclusively within the same female matrilines across multiple generations (Chesser 1991a,b). In C. sphinx, juveniles of both sexes dispersed from their natal harems (Storz et al. 2000b) and there were no detectable differences in allelic frequencies between the sexes (Storz et al. 2001b). Strongly negative values of $F_{\mathrm{IS}}$ in offspring cohorts are most likely attributable to the extremely high degree of polygyny.

Values of $F_{\text {IS }}$ accurately reflect population breeding structure insofar as the units of sampling represent the lowest hi- erarchical level of population structure at which random mating prevails (Long 1986; Sugg et al. 1996). When units of sampling constitute amalgamations of functionally independent reproductive units, interpretation of $F_{\mathrm{IS}}$ is potentially confounded by a Wahlund effect whereby genetic variance between reproductive subgroups produces a heterozygote deficit relative to Hardy-Weinberg expectations for the group as a whole. Positive values of $F_{\mathrm{IS}}$ that arise as an artifact of sampling design are problematic in genetic studies of human populations when units of sampling are hierarchical subdivisions of tribes that do not accurately reflect the true network of marital ties (Workman and Niswander 1970; Neel and Ward 1972; Long 1986). Similar situations may arise in societies of nonhuman animals as a result of reproductive substructuring within social units. There was no evidence for a Wahlund effect in colonies of $C$. sphinx, as values of $F_{\text {IS }}$ (offspring) were identical to those of the constituent harems. This indicates that patterns of mating do not depart from random expectations even when harems in the same colony are analyzed as a collective unit.

Interpretation of $F_{I T}$ - In offspring cohorts of C. sphinx, negative values of $F_{\mathrm{IT}}$ indicate negative correlations between alleles within individuals relative to the allelic array of the total cohort. In both years, $F_{\mathrm{IT}}$ (offspring) was less than $F_{\mathrm{ST}}$ (adults), indicating that correlation of uniting gametes was more negative than expected on the basis of chance alone. Although this may reflect active inbreeding avoidance, such a pattern can also result from purely random mating in combination with dispersal of one or both sexes (Chesser 1991a,b). In the 1997 offspring cohort, the strongly negative $F_{\mathrm{IS}}$ and high $F_{\mathrm{ST}}$ were nearly equal in magnitude, such that population-wide genotypic frequencies conformed to panmictic proportions (in accordance with the relationship $\left.\left[1-F_{\mathrm{IT}}\right]=\left[1-F_{\mathrm{ST}}\right]\left[1-F_{\mathrm{IS}}\right]\right)$. In the 1998 cohort, however, the strongly negative $F_{\text {IS }}$ was not counterbalanced by a similarly extreme degree of subdivision (due to the more uniform distribution of paternity among groups; Storz et al. 2001b). Consequently, $F_{\text {IT }}$ was significantly negative only in the 1998 cohort (Table 3). $F_{\mathrm{IT}}$-values for the adult age-class revealed no exceptionally strong or consistent evidence for nonrandom mating, despite the slight population-wide heterozygote deficit in 1998.

\section{Generational Differences in F-statistics}

Biologically meaningful interpretation of $F$-statistics requires identification of the age-structure of breeding groups in relation to cycles of dispersal and reproduction. If sampling units comprise a heterogeneous mixture of pre- and postdispersal age classes, the partitioning of genetic variance within and among groups will not accurately reflect genetic structure of the Mendelian population (sensu Hartl and Clark 1997, p. 72). It is the genetic structuring of locally interbreeding units that is relevant to the dynamics of microevolutionary change. When units of population sampling are transient aggregations of close kin (e.g., a pooled sample of adults and offspring that will later disperse to breed elsewhere), resulting patterns of genetic structure 
Table 5. Generational differences in the partitioning of genetic variance in populations of six mammalian species. Genetic structuring was assessed relative to socially or spatially defined breeding units. Parental and filial generations are denoted by $t$ and $t+1$, respectively. For the two studies of human populations, generations are defined on the basis of age categories (see Spielman et al. 1977; Long 1986). For C. ludovicianus, $P$. hastatus, C. sphinx, and A. seniculus, F-statistics for the parental generation are reported for adult females only

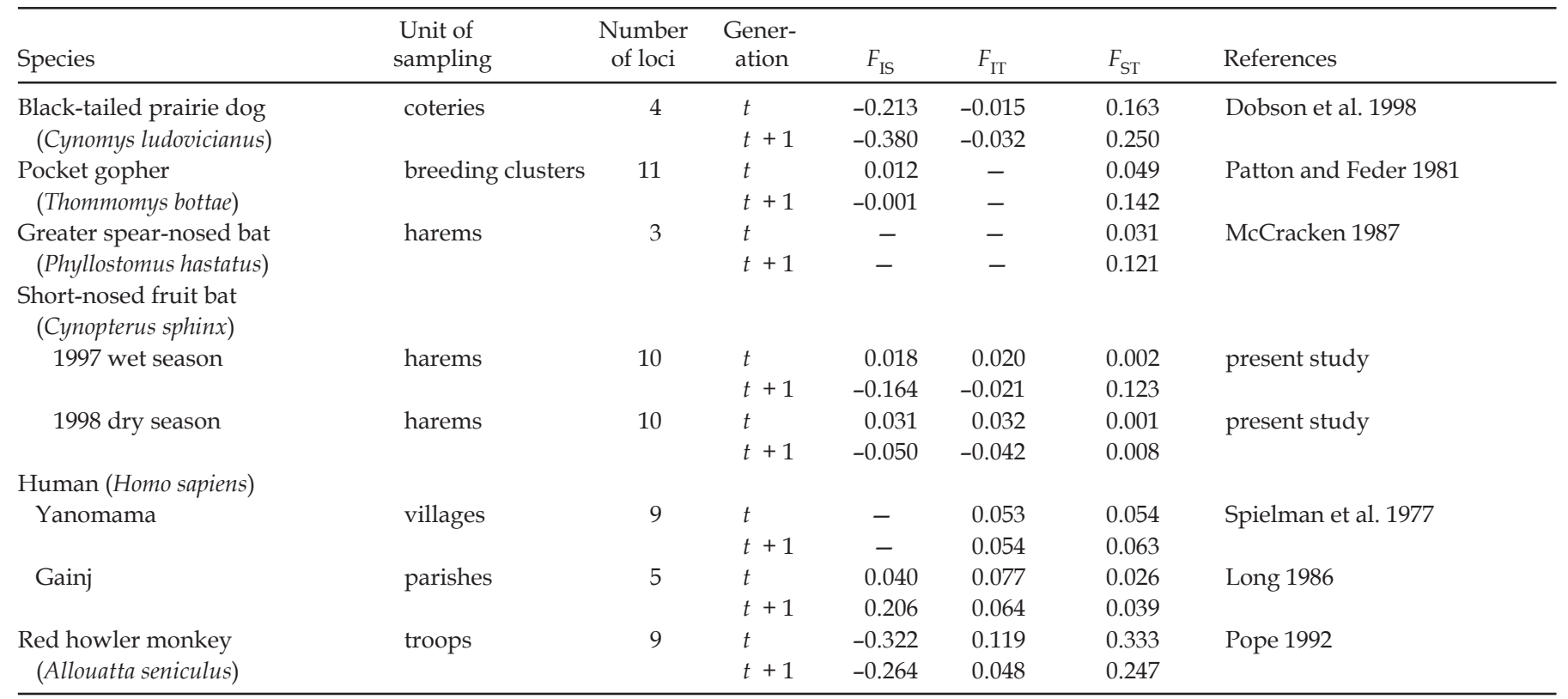

may have little evolutionary relevance. Although age structure may be a seriously confounding influence when it goes unrecognized, genetic analysis of discrete age-cohorts can provide a powerful mode of inference about the interplay between drift and gene flow.

In harem-forming species, the ability of individual males to monopolize mating access to groups of females results in high levels of relatedness within each harem offspring cohort. If genetic structuring of offspring cohorts is randomized by natal dispersal prior to recruitment into the breeding population, $F_{\mathrm{ST}}$ in the filial generation is expected to exceed $F_{\mathrm{ST}}$ in the parental generation. Such a pattern is clearly evident in the $C$. sphinx study population (Table 3). The generational disparity in $F_{\mathrm{ST}}$ was especially pronounced in 1997, as the wet-season cohort of offspring was characterized by the highest variance in representation of male parents (Storz et al. 2001b). A similar pattern is evident in populations of several other mammalian species where F-statistics have been estimated for pre- and postdispersal age classes (Table 5). With the exception of red howler monkeys, $F_{\mathrm{ST}}$ among groups composed of offspring only were invariably higher than $F_{\mathrm{ST}}$ among the same groups composed of adults only. Generational differences in $F_{\mathrm{ST}^{-}}$-values were most pronounced in C. sphinx (in 1997), greater spear-nosed bats (Phyllostomus hastatus), and pocket gophers (Thomomys bottae). These species are each characterized by female-biased sex ratios in breeding units, high variance in male mating success, and female dispersal (McCracken and Bradbury 1981; Patton and Feder 1981; Daly and Patton 1990; Storz et al. 2000b). The observed set of Fstatistics for the C. sphinx study population clearly support a mode of population structure characterized by polygynous outbreeding (or nonassortative mating with respect to kinship) and female dispersal.

\section{Drift and Inbreeding in Socially Structured Populations}

Recently it has been suggested that populations of social mammals may be characterized by effective sizes in excess of the adult census number ( $N$; Sugg et al. 1996). In a subdivided population, only the within-group component of genetic variance is subject to the sampling effects of Mendelian segregation in heterozygotes. With restricted dispersal, the resultant decrease in within-group variance (and the concomitant increase in among-group variance) impedes the rate of loss of variation from the population as a whole (Chesser et al. 1993; Wang 1997). Because the among-group component of variance is safeguarded from sampling drift, $N_{e}$ is larger than would be the case if random mating prevailed (Wright 1943; Sugg et al. 1996).

However, there are some important ecological factors to consider when assessing the general applicability of this model to socially structured populations. For example, effects of population substructure and local inbreeding on $N_{e}$ depend on the hierarchical level at which population numbers are most strongly regulated (Whitlock and Barton 1997; Nunney 1999). In the absence of local density regulation (the interdemic model of Nunney 1999), variance in productivity of breeding groups produces an additional source of drift and reduces $N_{e}$ relative to expectations for a panmictic population with the same census number (Whitlock and Barton 1997; Nunney 1999; Wang and Caballero 1999). When interdemic drift acts in concert with local inbreeding, increasing either $F_{\mathrm{IS}}$ or $F_{\mathrm{ST}}$ reduces the effective size of the total population. Because there were no strong or consistent departures from panmictic expectations in the adult gene pool of C. sphinx and no additional source of drift was associated with the process of group turnover, we conclude that genetic substructure has no significant effect on the effective size of the study population. 


\section{Implications for Social Evolution}

In harem-forming mammals, polygynous mating may promote high levels of paternal relatedness conducive to the expression of cooperative and altruistic behaviors within age cohorts (Altmann 1979). In such cases, expression of kin-selected behaviors among same-age individuals may be expected to take precedence over age-asymmetric behaviors such as those involved in cooperative breeding systems (e.g., when nonreproductive individuals aid in the rearing of younger siblings). However, high rates of natal dispersal and/or low rates of juvenile survivorship may preclude continued interaction among half-sibling progeny beyond the prereproductive maturation phase. This seems to be the case in C. sphinx. Despite high levels of paternal relatedness among harem offspring (particularly those born in the wet-season parturition period), there would seem to be little scope for continued interaction among half-sibling juveniles due to complete natal dispersal and low rates of juvenile survivorship (Storz et al. 2000b). The relatedness structure of offspring cohorts is therefore highly ephemeral. Low relatedness among harem females indicates that kin selection is an unlikely explanation for the formation and maintenance of behaviorally cohesive breeding groups in this highly social mammal.

\section{Acknowledgments}

We thank D. N. Deobagkar, S. R. R. Reddy, S. L. Shinde, and R. Patel for logistical help with fieldwork and M. Lara, C. J. Schneider, and J. Worthington Wilmer for technical assistance in the lab. We also thank G. F. McCracken for sharing Pteropus primer sequences and C. J. Schneider, B. J. Crespi, and two anonymous reviewers for constructive comments on the manuscript. This study was funded by grants to JFS and THK from the National Geographic Society and the Lubee Foundation and grants to JFS from the National Science Foundation (Dissertation Improvement Grant DEB 97-01057), Sigma Xi, the American Society of Mammalogists, Bat Conservation International, and the Society for Integrative and Comparative Biology. JFS received additional support from the Albert R. and Alma Shadle Fellowship in Mammalogy.

\section{Literature Cited}

Altmann J. 1979. Age cohorts as paternal sibships. Behav. Ecol. Sociobiol. 6:161-164.

Balasingh J., J. Koilraj, and T. H. Kunz. 1995. Tent construction by the short-nosed fruit bat, Cynopterus sphinx (Chiroptera: Pteropodidae) in southern India. Ethology. 100:210-229.

Barton N. H., and M. C. Whitlock. 1997. The evolution of metapopulations. Pp. 183-210 in I. Hanski and M. E. Gilpin, eds. Metapopulation biology: ecology, genetics, and evolution. Academic Press, San Diego, CA.

Bhat H. R., and T. H. Kunz. 1995. Altered flower/fruit clusters of the kitul palm used as roosts by the short-nosed fruit bat, Cynopterus sphinx (Chiroptera: Pteropodidae). J. Zool. (Lond.). 235:597-604.

Bradbury J. W., and S. L. Vehrencamp. 1976. Social organization and foraging in emballonurid bats. I. Field studies. Behav. Ecol. Sociobiol. 1:337-381.
Bush G. L., S. M. Case, A. C. Wilson, and J. L. Patton. 1977. Rapid speciation and chromosomal evolution in mammals. Proc. Natl. Acad. Sci. USA. 74:3942-3946.

Chepko-Sade B. D., and W. M. Shields. 1987. The effects of dispersal and social structure on effective population size. Pp. 287322 in B. D. Chepko-Sade and Z. T. Halpin, eds. Mammalian dispersal patterns: the effects of social structure on population genetics. Univ. of Chicago Press, Chicago, IL.

Chesser R. K. 1991a. Gene diversity and female philopatry. Genetics. 127:437-447.

Chesser R. K. 1991b. Influence of gene flow and breeding tactics on gene diversity within populations. Genetics. 129:573-583.

Chesser R. K., O. E. Rhodes Jr.,, D. W. Sugg, and A. Schnabel. 1993. Effective sizes for subdivided populations. Genetics. 135:1221-1232.

Clutton-Brock T. H. 1989. Female transfer and inbreeding avoidance in social mammals. Nature. 337:70-72.

Daly J. C., and J. L. Patton. 1990. Dispersal, gene flow, and allelic diversity between local populations of Thomomys bottae pocket gophers in the coastal ranges of California. Evolution. 44:1283-1294.

Dobson F. S. 1982. Competition for mates and predominant juvenile male dispersal in mammals. Anim. Behav. 30:1183-1192.

Dobson F. S., R. K. Chesser, J. L. Hoogland, D. W. Sugg, and D. W. Foltz. 1998. Breeding groups and gene dynamics in a socially structured population of prairie dogs. J. Mammal. 79:671-680.

Goodnight K. F., and D. C. Queller. 1999. Computer software for performing likelihood tests of pedigree relationship using genetic markers. Mol. Ecol. 8:1231-1234.

Goudet J. 1995. FSTAT (version 1.2): a computer program to calculate F-statistics. J. Hered. 86:485-486.

Goudet J., M. Raymond, T. de Meeüs, and F. Rousset. 1996. Testing differentiation in diploid populations. Genetics. 144:1933-1940.

Greenwood P. J. 1980. Mating systems, philopatry, and dispersal in birds and mammals. Anim. Behav. 28:1140-1162.

Guo S. W., and E. A. Thompson. 1992. Performing the exact test of Hardy-Weinberg proportion for multiple alleles. Biometrics. 48:361-372.

Hartl D. L., and A. G. Clark. 1997. Principles of population genetics. Sinauer Associates, Sunderland, MA.

Long J. C. 1986. The allelic correlation structure of Gainj- and Kalam-speaking people. I. The estimation and interpretation of Wright's F-statistics. Genetics. 112:629-647.

McCracken G. F. 1987. Genetic structure of bat social groups. Pp. 281-298 in M. B. Fenton, P. Racey, and J. M. V. Rayner, eds. Recent advances in the study of bats. Cambridge Univ. Press, Cambridge, U.K.

McCracken G. F., and J. W. Bradbury. 1981. Social organization and kinship in the polygynous bat Phyllostomus hastatus. Behav. Ecol. Sociobiol. 8:11-34.

Michod R. E. 1993. Inbreeding and the evolution of social behavior. Pp. 74-96 in N. H. Thornhill, ed. The natural history of inbreeding and outbreeding. Univ. of Chicago Press, Chicago, IL.

Neel J. V., and R. H. Ward. 1972. The genetic structure of a tribal population, the Yanomama Indians. VI. Analysis by F-statistics including a comparison with the Makiritire and Xavante. Genetics. 72:639-666. 
Nunney L. 1999. The effective size of a hierarchically structured population. Evolution. 53:1-10.

Patton J. L., and J. H. Feder. 1981. Microspatial genetic heterogeneity in pocket gophers: non-random breeding and drift. Evolution. 35:912-920.

Pope T. R. 1992. The influence of dispersal patterns and mating system on genetic differentiation within and between populations of the red howler monkey (Alouatta seniculus). Evolution. 46:1112-1128.

Prout T. 1981. A note on the island model with sex dependent migration. Theor. Appl. Genet. 59:327-332.

Pusey A. E. 1987. Sex-biased dispersal and inbreeding avoidance in birds and mammals. Trends Ecol. Evol. 2:295-299.

Pusey A. E., and C. Packer. 1987. Philopatry and dispersal. Pp. 250-266 in B. B. Smuts, D. L. Cheney, R. M. Seyfarth, T. T. Struhsaker, and R. W. Wrangham, eds. Primate societies. Univ. of Chicago Press, Chicago, IL.

Pusey A. E., and M. Wolf. 1996. Inbreeding avoidance in animals. Trends Ecol. Evol. 11:201-206.

Queller D. C., and K. F. Goodnight. 1989. Estimating relatedness using genetic markers. Evolution. 43:258-275.

Raymond M., and F. Rousset. 1995a. An exact test for population differentiation. Evolution. 49:1280-1283.

Raymond M. 1995b. GENEPOP (Version 1.2): Population genetics software for exact test and ecumenicism. J. Hered. 86:248-249.

Smith A. T. 1993. The natural history of inbreeding and outbreeding in small mammals. Pp. 329-351 in N. H. Thornhill, ed. The natural history of inbreeding and outbreeding. Univ. of Chicago Press, Chicago, IL.

Smouse P. E., V. J. Vitzthum, and J. V. Neel. 1981. The impact of random and lineal fission on the genetic divergence of small human groups: a case study among the Yanomama. Genetics. 98:179-197.

Sokal R. R., and F. J. Rohlf. 1995. Biometry. W. H. Freeman and Company, New York.

Spielman R. S., J. V. Neel, and F. H. F. Li. 1977. Inbreeding estimation from population data: models, procedures, and implications. Genetics. 35:355-371.

Storz J. F. 1999. Genetic consequences of mammalian social structure. J. Mammal. 80:553-569.

Storz J. F. 2000. Variation at tri- and tetranucleotide repeat microsatellite loci in the fruit bat genus Cynopterus (Chiroptera: Pteropodidae). Mol. Ecol. 9:2198-2200.

Storz J. F., and T. H. Kunz. 1999. Cynopterus sphinx. Mammal. Species. 613:1-8.

Storz J. F., J. Balasingh, P. T. Nathan, K. Emmanuel, and T. H. Kunz. 2000a. Dispersion and site-fidelity in a tent-roosting population of the short-nosed fruit bat (Cynopterus sphinx) in southern India. J. Trop. Ecol. 16:117-131.

Storz J. F., H. R. Bhat, and T. H. Kunz. 2000b. Social structure of a polygynous tent-making bat, Cynopterus sphinx (Megachiroptera). J. Zool. (Lond.). 251:151-165.

Storz J. F., J. Balasingh, H. R. Bhat, P. T. Nathan, A. Prakash, D. P. Swami Doss, and T. H. Kunz. 2001a. Clinal variation in body size and sexual dimorphism in an Indian fruit bat, Cynopterus sphinx (Chiroptera: Pteropodidae). Biol. J. Linn. Soc. 72:17-31.

Storz J. F., H. R. Bhat, and T. H. Kunz. 2001b. Genetic consequences of polygyny and social structure in an Indian fruit bat, Cynopterus sphinx. II. Variance in male mating success and effective population size. Evolution 55:1224-1232.

Sugg D. W., R. K. Chesser, F. S. Dobson, and J. L. Hoogland. 1996. Population genetics meets behavioral ecology. Trends Ecol. Evol. 11:338-342.

Uyenoyama M. K. 1984. Inbreeding and the evolution of altruism under kin selection: effects on relatedness and group structure. Evolution. 48:778-795.

Wade M. J. 1996. Adaptation in subdivided populations: kin selection and interdemic selection. Pp. 381-405 in M. R. Rose and G. V. Lauder, eds. Adaptation. Academic Press, San Diego, CA.

Wade M. J., and F. J. Breden. 1987. Kin selection in complex groups: mating structure, migration structure, and the evolution of social behaviors. Pp. 273-283 in B. D. Chepko-Sade and Z. T. Halpin, eds. Mammalian dispersal patterns: the effects of social structure on population genetics. Univ. of Chicago Press, Chicago.

Wade M. J., and C. J. Goodnight. 1998. The theories of Fisher and Wright in the context of metapopulations: when nature does many small experiments. Evolution. 52:1537-1553.

Wang J. 1997. Effective size and F-statistics of subdivided populations. II. Dioecious species. Genetics. 146:1465-1474.

Wang J., and A. Caballero. 1999. Developments in predicting the effective size of subdivided populations. Heredity. 82:212-226.

Weir B. S., and C. C. Cockerham. 1984. Estimating F-statistics for the analysis of population structure. Evolution. 38:1358-1370.

Whitlock M. C. 1992. Nonequilibrium population structure in forked fungus beetles: extinction, colonization, and the genetic variance among populations. Am. Nat. 139:952-970.

Whitlock M. C., and N. H. Barton. 1997. The effective size of a subdivided population. Genetics. 146:427-441.

Whitlock M. C., and D. E. McCauley. 1990. Some population genetic consequences of colony formation and extinction: genetic correlations within founding groups. Evolution. 44:1717-1724.

Wilkinson G. S. 1985a. The social organization of the common vampire bat. I. Pattern and cause of association. Behav. Ecol. Sociobiol. 17:111-121.

Wilkinson G. S. 1985b. The social organization of the common vampire bat. II. Mating system, genetic structure, and relatedness. Behav. Ecol. Sociobiol. 17:123-134.

Williams C. F. 1986. Social organization of the bat, Carollia perspicillata (Chiroptera: Phyllostomidae). Ethology. 71:265-282.

Wilson A. C., G. L. Bush, S. M. Case, and M. C. King. 1975. Social structuring of mammalian populations and the rate of chromosomal evolution. Proc. Natl. Acad. Sci. USA. 72:5061-5065.

Workman P. L., and J. D. Niswander. 1970. Population studies on southwestern Indian tribes. Am. J. Hum. Genet. 22:24-49.

Wright S. 1922. Coefficients of inbreeding and relationship. Am. Nat. 56:330-338.

Wright S. 1943. Isolation by distance. Genetics. 28:114-138.

Wright S. 1951. The genetical structure of populations. Ann. Eugen. 15:323-354.

Wright S. 1965. The interpretation of population structure by Fstatistics with special regard to the system of mating. Evolution. 19:395-420. 